\title{
Outcome Measures in Medication Trials for Substance Use Disorders
}

David J. McCann, Ph.D Tatiana Ramey, M.D, Ph.D Phil Skolnick, Ph.D., D.Sc.

\section{Address}

*Division of Pharmacotherapies and Medical Consequences of Drug Abuse, National Institute on Drug Abuse, National Institutes of Health, 6001 Executive Boulevard, Bethesda, MD 20892-9551, USA

Email: phil.skolnick@nih.gov

Published online: 11 April 2015

(C) Springer International Publishing AG (outside the USA) 2015

This article is part of the Topical Collection on Substance Use Disorders

Keywords Addiction - Substance use disorders - Pharmacotherapies - Abstinence $\cdot$ Drugs of abuse · Clinical endpoints

\section{Opinion statement}

There are multiple therapeutic options to treat tobacco (e.g., nicotine replacement therapies, varenicline) and alcohol (e.g., naltrexone, acamprosate) use disorders. In contrast, there are currently no FDA-approved pharmacotherapies to treat stimulant (e.g., cocaine, methamphetamine) use disorders. Based on a commentary published by FDA staff, a period of sustained abstinence appears required for regulatory approval of a first-in-class medication to treat stimulant use disorders. Certainly, achieving abstinence remains the goal of treatment in both real world medical practice and clinical trials. However, if a medication can help patients to significantly reduce stimulant use (short of achieving a sustained abstinence) while attempting to quit, such reductions could confer meaningful benefit. The FDA has adopted the "percentage of subjects with no heavy drinking days" as an endpoint for pharmacotherapy trials in alcohol use disorders; this suggests there may be a potential path forward for developing analogous, non-abstinence endpoints for stimulant use disorder trials. However, reductions in drug use per se (short of abstinence) must have prognostic value in order to be considered an acceptable basis for FDA approval. Thus, even if a medication can provide sustained reductions in drug use, the challenge ahead is to demonstrate that this "success" is accompanied by benefits that accrue in dimensions readily understood by patients and their families, and of sufficient value to be reimbursed by third party payers. Emerging data sets discussed in this paper indicate that endpoints other than abstinence may ultimately be validated as outcome measures in pharmacotherapy trials for stimulant use disorders. 


\section{Introduction}

Private sector investment in the development of medications to treat substance use disorders (SUDs) is modest, particularly when compared to the societal costs created bythesedisorders.Forexample, morethan $20 \%$ oftheadult US population currently smokes (http://www.samhsa. gov/atod/tobacco), which translates to a potential market ofmorethan 60 millionindividuals. Nonetheless, onlyone novel medication (varenicline; Chantix ${ }^{\circledR} /$ Champix $^{\circledR}$ ) has reached the market as a smoking cessation aid in the past decade. Farmoreinvestmentgoesinto the development of pharmacotherapies to treat the consequences of smoking (e.g., lung cancer, COPD) compared with the proximal cause [1]. While psychiatric drug development has been in retreat for almost a decade [2,3], the lack of private sector investment in the development of medications to treat SUDs has long been acknowledged as a problem [4]. The perception of a very high regulatory "bar" for approval is among the multiple factors that have contributed to this lack of investment [5]. In this article, we will review and discuss the current state of outcome measures in medicationtrialsforSUDs. Forillicitdrugs, ourfocuswillbelimited to cocaineand methamphetamine dependence, two highpriority disorders in need of first-in-class pharmacotherapies.

\section{Pharmacotherapy trials in alcoholism}

For alcohol use disorder, the FDA currently recognizes the percent of subjects with no heavy drinking days (PSNHDDs) as a primary endpoint measure in medication trials $[6,7]$. This is essentially a binary (dichotomous) outcome, with no heavy drinking days considered a success, and $\geq 1$ heavy drinking day(s) a failure. Based on this outcome measure, an effective pharmacotherapy would result in a significantly higher percentage of subjects reporting no heavy drinking days, defined as $\geq 4$ drinks/day for women and $\geq 5$ drinks/day for men, respectively, the current NIAAA definition of high-risk drinking [6]. The basis for this endpoint is the evidence linking heavy drinking days both to negative medical (e.g., liver and cardiovascular disease) and psychosocial (marital issues, loss of driver's license) consequences [6]. Furthermore, a low level of alcohol consumption (one drink/day for women, two drinks/day for men) has been linked to salutary outcomes (e.g., cardiovascular function) [8], and many patients who do not wish to quit may more readily embrace the goal of reduced drinking rather than total abstinence.

The FDA has also endorsed a "grace period" for pharmacotherapy trials in SUDs, initially for alcohol and tobacco studies (e.g., 7, 9) and subsequently for other SUDs, based on a commentary $[10 \bullet \bullet]$ that accompanied the reanalysis of

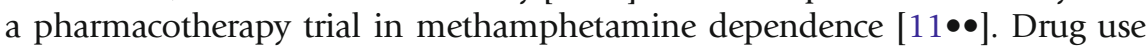
during a grace period can be excluded from the efficacy analysis. This grace period (of unspecified duration) may allow medications to reach effective levels, which could be particularly important in medications that require lengthy titration period to minimize side effects. In the case of medications that block the rewarding effects of abused substances, a grace period may allow time for learning and associated behavioral adaptation. Perhaps most important, a grace period may begin the process of correcting the neurochemical dysregulation created by years of drug dependence [12], which in turn may result in patients more able to engage in treatment. While a fixed duration grace period is often utilized in a dichotomized, success/failure outcome [6], the use of a flexible grace period can increase statistical power $[11 \bullet \bullet]$. 
Reanalysis of two large multisite alcohol trials using PSNHDDs as the outcome measure together with a grace period [6] resulted in statistically significant medication effects (naltrexone and topiramate) with small (0.22$0.26)$ Cohen's effect sizes. The PSNHDDs for naltrexone and placebo were 44 and $33.4 \%$, respectively, while the PSNHDDs in the topiramate study for drug and placebo were 13.4 and $5.9 \%$, respectively. Permitting a "slip" (one heavy drinking day) after the grace period did not remarkably change the effect size or differences in the PSNHDDs between medication and placebo groups. However, compared to subjects with one heavy drinking day (HDD), subjects with no HDDs during the last 2 months of the COMBINE study (comparing oral naltrexone and placebo in this instance) had a significantly lower risk of alcohol-related consequences, as well as lower levels of all drinking outcome measures (including drinks/day and \% days abstinent) during follow-up for as long as 1 year after the end of treatment. Thus, when combined with a grace period that forgives slips during the initial period of pharmacotherapy, PSNH DDs represent an endpoint that is more realistic and achievable than complete abstinence, and is an outcome that has already been achieved by available medications.

\section{Pharmacotherapy trials in tobacco dependence}

In the absence of compelling data to suggest either salutary health benefits of an abused substance or that a reduction in use (short of achieving abstinence) confers some recognizable benefits to patients, abstinence appears to be the

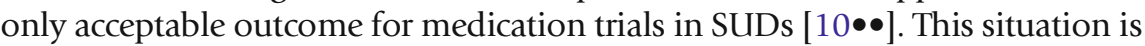
exemplified by tobacco dependence, since no amount of tobacco consumption appears to confer benefit, and while individuals who remain abstinent from tobacco derive long-term health benefits [13], individuals who only reduce their level of smoking have not been shown to achieve meaningful benefit. Correspondingly, the increase in subjects achieving sustained abstinence was used as the basis for approval of varenicline [9]. With multiple smoking cessation products already on the market, a high bar was set for the approval of varenicline: not only was a significant increase in abstinence rate (compared to placebo) required at the end of the 12-week treatment period, maintenance of a significant separation from placebo was also required at study week 52 (that is, nine months after treatment discontinuation). It seems unlikely that such a high bar would have been set if varenicline had been a first-in-class medication. Such long-term data were not required for approval of nicotine replacement therapies or bupropion $\left(\right.$ Zyban $\left.^{\circledR}\right)$.

\section{Pharmacotherapy trials in cocaine and methamphetamine dependence}

In the absence of either FDA guidelines or any approved medications that could serve as benchmarks, it is logical for cocaine and methamphetamine dependence clinical trial designs to be shaped by experience in other SUDs. However, there are important differences between licit and illicit substances that are 
integral to a discussion of outcome measures. When a patient smokes a cigarette or drinks an alcoholic beverage, the product is fairly consistent and drug intake can be quantified (for example, the number of beers consumed with defined alcohol content). Moreover, there is virtually no concern about immediate death due to smoking or alcohol ingestion. A patient buying "cocaine" or "methamphetamine" on the street, however, is administering a product of unknown purity that may contain dangerous adulterants, and there is a risk of serious harm from the acute ingestion of the intended substance or other chemicals. Further, behaviors related to the act of using and obtaining illicit drugs (such as needle sharing, purchasing, and illegal activities associated with obtaining the monies to purchase) all expose an individual to risks not shared by alcohol and tobacco. Thus, when weighing the value of similar reductions in licit and illicit substance use, it can be argued that short-term reductions in illicit substance use are substantially more beneficial to patients.

Abstaining from illicit substances is a pattern of use that is generally held to

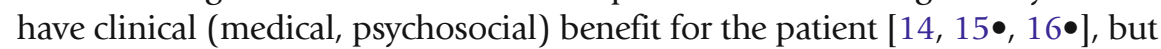
what duration of abstinence must be achieved to infer meaningful benefit? From an intuitive standpoint, any reduction in the frequency an individual engages in such risky behavior would appear to benefit the patient. If taking an illicit drug such as cocaine is viewed as playing Russian roulette, then reducing the frequency that an individual engages in this behavior can, in toto, be viewed as taking fewer turns spinning the cylinder. For alcohol and tobacco dependence, the value of quitting for 3 or 4 weeks at the end of an 8 to 12 week clinical trial, without evidence of maintained abstinence in long-term followup visits, is questionable. In contrast, for illicit drugs, 3 or 4 weeks of abstinence may have critical value for the patient. While such periods of brief abstinence may also hold the promise of long-term abstinence (a lifetime of abstinence from cocaine must begin with 3 weeks), requiring long-term abstinence as an endpoint for regulatory approval of a first-in-class medication may be setting the bar too high.

With the FDA's adoption of PSNHDDS as an endpoint for alcohol trials, the potential for development of analogous non-abstinence endpoints for illicit drugs merits consideration. Because of the serious health risks associated with each use of an illicit substance as well as the risk of arrest and incarceration that accompanies continued use, abstinence appears to be the gold standard that treatment providers can hold up for patients. Thus, in real world medical practice and clinical trials, achieving abstinence should continue to be the goal of treatment. However, if a medication can help patients to significantly reduce (e.g.) cocaine use (short of achieving abstinence) while they are attempting to quit, this could confer meaningful benefit.

\section{Alternative outcome measures/endpoints for medication trials in SUDs}

Multiple clinical studies have demonstrated that pharmacotherapies can produce statistically significant reductions in illicit drug use. These studies have used both continuous and discontinuous measures of drug use $[15 \bullet, 17]$ to demonstrate these effects, including the percent of drug-free urine samples over 
the course of a trial [18], weeks of abstinence and/or longest periods of continuous abstinence during the trial $[19,20]$ and weekly fraction of cocaine non-use days $[20,21]$. Nonetheless, as described in the previous section, reductions in drug use per se (short of achieving abstinence) must have prognostic value in

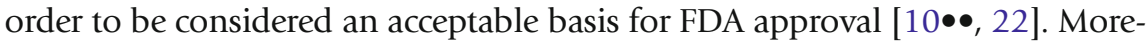
over, even if a medication is capable of sustaining such reductions in drug use, then this "success" must be accompanied by benefits which accrue in a dimension(s) which will be readily understood by patients (and their families), relevant for public health policy makers, and of sufficient value to receive reimbursement from third party payers.

Linking reductions in drug use with clinically meaningful benefits is a formidable challenge, perhaps more so within the confines of pharmacotherapy trials of relatively short duration (typically, 8-12 weeks) that often do not include long-term follow-up. There are, however, emerging data sets indicating that endpoints other than abstinence may ultimately fulfill the high hurdle of impact on consequences expressed in the Winchell et al. (2012) commentary.

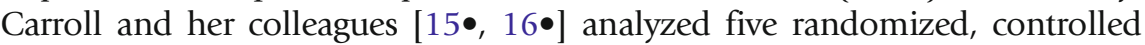
clinical trials (which included medication (disulfiram) and in one case, behavioral therapy) in cocaine-dependent subjects $(n=434)$. Several continuous (e.g., percent days abstinent, percent negative urine samples, maximum days of continuous abstinence) and dichotomous (abstinence for $\geq 21$ consecutive days during the trial) outcome measures frequently used in clinical trials were reported as significantly correlated (albeit with $r$ values rarely exceeding 0.3) with both cocaine use and "good functioning" (an ASI-based composite of no cocaine use, together with no reported legal, psychological, family, or employment problems for the preceding 28 days) during the follow-up period. That is, measures such as $\%$ of subjects attaining $3+$ weeks of abstinence during the trial were significantly (albeit with $r$ values of $\sim 0.25$ ) correlated with abstinence throughout follow-up and good functioning (as described above) at follow-up months 1, 3, 6, and 12. These data raise some potential areas for further exploration in future pharmacotherapy trials. The relatively low $r$ values linking the reductions in drug taking with improvement in consequences at follow-up are perhaps not surprising, and consistent with the view of Martin et al. [23] on the difficulties in measurement and methodological issues associated with quantitating the consequences of reductions in drug use. Given the complexities in measuring the distal consequences of reduced drug use (also discussed in 22), these new results represent an important step in the right direction. Thus, additional studies using different medications capable of achieving one or more of the same abstinence-related endpoints described by Carroll et al. [15•] and Kiluk et al. [16•] would lend itself to a similar "good functioning" analysis using the "global problems" modeling construct that appears more sensitive than ASI domains composite scores in the above mentioned studies. A success/ failure dichotomization of this good functioning outcome during the follow-up period could arguably represent a clinically meaningful outcome based on a patient achieving no cocaine use and a lack of problems in multiple psychosocial domains. Integrating this "good functioning" composite with a pharmacoeconomic analysis with, for example, an outcome demonstrating a reduced burden on state/federal resources could make such a medication more attractive to third party payers. Carroll et al. [15•] also reported that dichotomized versions of reduced frequency of drug use (in this analysis, $50 \%$ or $75 \%$ 
reductions) were found not to be sufficiently strongly related to indicators of either cocaine use or functioning (as described above) during follow-up. These authors suggest that the susceptibility of reduced use measurements to missing data and the difficulty in biological verification of drug use may contribute to the insensitivity of the measure. In a complementary analysis of the same dataset using structural equation modeling, Kiluk et al. [16•] reported that higher levels of cocaine abstinence (using both continuous and discontinuous outcomes) during the trial (not necessarily end-of-study abstinence) were associated with fewer reported global problems (as described above) at both end of treatment and follow-up. By including "days of problems" from ASI areas related to consequences of drug use in their global latent construct model, the authors indicate model sensitivity was improved, and also recommend the dichotomous measure of $\geq 21$ days of continuous abstinence as a primary endpoint measure in medication trials. These authors conclude that the analyses indicate abstinence achieved during treatment is associated with fewer addiction-related problems when treatment ends. However, ASI subscales and the ASI composite score per se lack sensitivity; the creation of a "days of global problems" construct was used to improve sensitivity [16•], which could be important for both regulatory and reimbursement purposes. In this context, it is useful to mention the commentary by Martin et al. [23], challenging the notion that psychosocial and other societal consequences of SUDs belong to its core diagnosis. These authors point to the methodological, conceptual, and measurement issues, concluding that consequences of drug use should be regarded at best as ancillary measures. They emphasize the low sensitivity and specificity, measurement issues, and overlaps in how consequences are determined, as well as the frequent lack of direct causality of these outcomes.

The search for associations of reduced drug use with distal consequences as a means of evaluating both reductions in use and abstinence-oriented, longerterm outcomes was also examined by Crits-Christoph et al. [17]. These investigators analyzed data from the NIDA cocaine collaborative treatment study consisting of 487 patients diagnosed with DSM-IV cocaine dependence. Patients were treated with psychosocial interventions, assessed monthly during the 6-month treatment period, then at 3-month intervals up to 18 months of follow-up. The authors reported that the strongest associations were found between abstinence related measures (both continuous and discontinuous) and abstinence at follow up (12 months). Although there were multiple statistically significant correlations, the absolute values of the partial correlations were low (0.15-0.3 range), consistent with the findings reported in the Carroll et al. [15•] data set. There were also statistically significant, albeit small correlations between some of the reduction in use (both abstinence and reduction) measures and the ASI legal subscale at month 12. While these studies were focused on psychosocial rather than medication based interventions, the data are generally consistent with the conclusions from the Carroll et al. [15•] and Kiluk et al. [16•] analyses, indicating that abstinence measured during the course of a trial is related to abstinence at long-term follow-up.

The association of reductions in cocaine use with health-related outcomes has been an elusive goal. Recently, Lai et al. [24•] described a pilot study consisting of 22 African American male chronic (average length of use $\sim 13$ years) cocaine users enrolled in a voucher incentive-based program. Subjects received escalating monetary rewards for sustaining abstinence from 
cocaine (confirmed by urinalysis), while a positive test reset the incentive level to week 1 . During a six-month period, mean use of cocaine was reduced between $\sim 60-80 \%$. These investigators reported (after adjusting for sex, age, and cardiovascular risk) that 6 months of cocaine abstinence was associated with lower circulating levels of endothelin-1 (ET-1). Moreover, the number of days of cocaine use was positively correlated with ET-1 levels. These associations, evinced using a generalized estimating equation analysis and small sample size, must be considered very preliminary. Nonetheless, the observation that cocaine abstinence and reduced use of cocaine are both associated with lower ET-1 levels is consistent with a previous report of significantly higher ET-1 levels in cocaine users compared to non-users, and that 1 month of abstinence in a drug rehabilitation setting resulted in significantly lower levels of ET-1 [25]. Because ET-1 is a marker for endothelial function and damage [26], changes in ET-1 may ultimately prove useful as a downstream biomarker for assessing salutary consequences of reductions in cocaine use. Thus, cocaine use has been implicated in the development and acceleration of coronary artery disease [27, $28]$, and both clinical and epidemiological evidence suggest that cocaine use is associated with subclinical atherosclerosis [29] perhaps leading to studies that could validate a health outcome measure for medications targeted at cocaine use disorder.

\section{Conclusion}

Reductions in cocaine use may be correlated with fewer problems in areas broadly connected to general health and psychosocial arenas/domains. While the reported correlations $[15 \bullet, 16 \bullet]$ are not robust, these data indicate that reduced drug use tips the point of risk-benefit in the desired direction. Additional data, likely to emerge from large epidemiological studies, will be required to corroborate and expand on these initial findings. Newly emerging data from Lai and his associates [24•] also offer the promise of a biomarker (circulating levels of endothelin-1) linked to a health-related outcome that appear sensitive to changes in cocaine use. In addition, with an ever increasing knowledge of neurocircuitry, the affective, motivational, cognitive, and social functions that constitute daily experience can be viewed as patterns of interactions among networked brain circuits. Symptoms and symptom domains (in this case, as related to SUDs) may then be conceptualized as alterations of neuronal networks. These alterations can be viewed as the foundations of susceptibility to domains of neuro- and psychopathology. On this basis, we may be on the brink of a new era, wherein place of discrete disorders defined by DSM-V criteria, symptom domain(s), potentially crossing several DSM categories, may essentially become a therapeutic target with endpoints that would quantify neurocircuitry dynamics and their corresponding behavioral outcomes, perhaps even across a range of SUDs and beyond [30,31]. Studies that conceptualize addictions within the framework of Research Domain Criteria (RDoC) defining symptom domains that associate brain circuitry and function may impact the structure of endpoints and redefine outcomes for pharmacotherapy trials in years to come. These types of data could form a foundation for public policy decisions as well as provide endpoints and outcome measures for pharmacotherapy trials in SUDs. 


\section{Compliance with Ethics Guidelines}

\section{Conflict of Interest}

David J. McCann declares that he has no conflict of interest.

Tatiana Ramey holds stock in Pfizer, Inc., and Eli Lilly Inc., unrelated to this article.

Phil Skolnick has received royalties from Current Protocols in Neuroscience and travel accommodations/ expense reimbursement from FASEB which are unrelated to this article. Dr. Skolnick holds stock in Eli Lilly, Merck and Bristol Myers Squibb, also unrelated to this article.

\section{Human and Animal Rights and Informed Consent}

This article does not contain any studies with human or animal subjects performed by any of the authors.

\section{References}

Papers of particular interest, published recently, have been

highlighted as:

- Of importance

$\bullet$ Of major importance

1. Pollock JD, Koustova E, Hoffman A, Shurtleff D, Volkow ND. Treatments for nicotine addiction should be a top priority. Lancet. 2009;374:513-4.

2. Fibiger HC. Psychiatry, the pharmaceutical industry, and the road to better therapeutics. Schizophr Bull. 2012;38:649-50.

3. Brady LS, Insel TR. Translating discoveries into medicine: psychiatric drug development in 2011. Neuropsychopharmacology. 2012;37:281-3.

4. The Development of Medication for the Treatment of Opiate and Cocaine Addictions. Washington, D.C., National Academy Press; 1995.

5. Acri JB, Skolnick P. Pharmacotherapy of substance use disorders. In: Charney D, Nestler E, Sklar P, Buxbaum J, editors. Neurobiology of mental illness. 4th ed. London: Oxford University Press; 2013. p. 235-45.

6. Falk D, Wang XQ, Liu L, Fertig J, Mattson M, Ryan $M$, et al. Percentage of subjects with no heavy drinking days: evaluation as an efficacy endpoint for alcohol clinical trials. Alcohol Clin Exp Res. 2010;34:2022-34.

7. Medical Review of Vivitrol. Rockville, MD: U.S. Government, 2006.

8. Mochly-Rosen D, Zakhari S. What did we learn from the French (paradox)? Alcohol Res Health. 2010;33:76-86.

9. Jorenby DE, Hays JT, Rigotti NA, Azoulay S, Watsky EJ, Williams KE, et al. Efficacy of varenicline, an alpha4beta2 nicotinic acetylcholine receptor partial agonist, vs placebo or sustained-release bupropion for smoking cessation: a randomized controlled trial. JAMA. 2006;296:56-63.

10.• Winchell C, Rappaport BA, Roca R, Rosebraugh CJ. Reanalysis of methamphetamine dependence treatment trial. CNS Neurosci Ther. 2012;18:367-8.
In the absence of published guidelines, this commentary provides a valuable insights on current FDA views on clinical trial endpoints for stimulant use disorders.

11.• McCann DJ, Li SH. A novel, nonbinary evaluation of success and failure reveals bupropion efficacy versus methamphetamine dependence: reanalysis of a multisite trial. CNS Neurosci Ther. 2012;18:414-8.

A novel analysis that weights the duration of end of study abstinence abstinence beyond a predefined minimum threshold of success. For example, the number of weeks a subject achieves abstinence beyond a set threshold of two weeks. This method appears to confer greater sensitivity than a traditional success/failure analysis.

12. Koob GF. Negative reinforcement in drug addiction: the darkness within. Curr Open Neurobiology. 2013;23:559-63.

13. Jha $\mathrm{P}$, Ramasundarahettige $\mathrm{C}$, Landsman $\mathrm{V}$, Rostron $\mathrm{B}$, Thun M, Anderson RN, et al. 21st-century hazards of smoking and benefits of cessation in the United States. N Engle J Med. 2013;368:341-50.

14. Scott CK, Dennis ML, Laudet A, Funk RR, Simeone RS. Surviving drug addiction: the effect of treatment and abstinence on mortality. Am J Public Health.

2011;101:737-44.

15. Carroll KM, Kiluk BD, Nich C, DeVito EE, Decker S, LaPaglia D, et al. Toward empirical identification of a clinically meaningful indicator of treatment outcome: features of candidate indicators and evaluation of sensitivity to treatment effects and relationship to one year follow up cocaine use outcomes. Drug Alcohol Depend. 2014;137:3-19.

This review uses pooled data from five randomized clinical trials of cocaine dependence to explore the sensitivities of multiple outcome measures and general functioning during follow-up. 
16. Kiluk BD, Nich C, Witkiewitz K, Babuscio TA, Carroll $\mathrm{KM}$. What happens in treatment doesn't stay in treatment: cocaine abstinence during treatment is associated with fewer problems at follow-up. J Consult Clin Psycho. 2014;82:619-27.

By modeling the data described in Carroll, et al. (15), these investigators provide evidence that within-treatment abstinence from cocaine provides benefit that extend beyond the frequency of drug use.

17. Crits-Cristoph P, Gallop R, Connolly Gibbons MB, Sadicario J, Woody G. Measuring outcome in the treatment of cocaine dependence. J Alcohol Drug Depend. 2013;1:1-8.

18. Jayaram-Lindstrom N, Hammarberg A, Beck O, Franck J. Naltrexone for the treatment of amphetamine dependence: a randomized, placebo-controlled trial. Am J Psychiatry. 2008;165:1442-8.

19. Mariani JJ, Pavlicova M, Bisaga A, Nunes EV, Brooks DJ, Levin FR. Extended-release mixed amphetamine salts and topiramate for cocaine dependence: a randomized controlled trial. Boil Psychiatry. 2012;72:950-6.

20. Johnson BA, Ait-Daoud N, Wang XQ, Penberthy JK, Javors MA, Seneviratne C, et al. Topiramate for the treatment of cocaine addiction: a randomized clinical trial. JAMA Psychiatry. 2013;70:1338-46.

21. Donovan DM, Bigelow GE, Brigham GS, Carroll KM, Cohen AJ, Gardin JG, et al. Primary outcome indices in illicit drug dependence treatment research: systematic approach to selection and measurement of drug use end-points in clinical trials. Addiction. 2012;107:694708.

22. Negus SS. Henning field J, Agonist medications for the treatment of cocaine use disorder.

Neuropsychopharmacology advanced online publication, 7 January 2015; doi:10.1038/npp2014.322
23. Martin CS, Langenbucher JW, Chung T, Sher KJ. Truth or consequences in the diagnosis of substance use disorders. Addiction. 2014;109:1773-8.

24. Lai H, Stitzer M, Treisman G, Moore R, Brinker J, Gerstenblith G, et al. Reduction in cocaine use is associated with less endothelial damage in African Americans: a preliminary study. J Addiction Med 2015.

Provides preliminary evidence that levels of endothelin-1 (ET-1) could represent a biomarker to examine longitudinal changes in cocaine use.

25. Saez CG, Olivares P, Pallavicini J, Panes O, Moreno N, Massardo T, et al. Increased number of circulating endothelial cells and plasma markers of endothelial damage in chronic cocaine users. Thromb Res. 2011;128:e18-23.

26. Chong AY, Blann AD, Lip GY. Assessment of endothelial damage and dysfunction: observations in relation to heart failure. QJM. 2003;96:253-67.

27. Kloner RA, Hale S, Alker K, Rezkalla S. The effects of acute and chronic cocaine use on the heart. Circulation. 1992;85:407-19.

28. Benzaquen BS, Cohen V, Eisenberg MJ. Effects of cocaine on the coronary arteries. Am Heart J. 2001;142:402-10.

29. Lai S, Lai H, Meng Q, Tong W, Vlahov D, Celentano D, et al. Effect of cocaine use on coronary calcium among black adults in Baltimore, Maryland. Am J Cardiol. 2002;90:326-8.

30. Cuthbert BN, Insel TR. Toward the future of psychiatric diagnosis: the seven pillars of RDoC. BMC Med. 2013;11:126.

31. Cuthbert BN. The RDoC framework: facilitating transition from ICD/DSM to dimensional approaches that integrate neuroscience and psychopathology. World Psychiatry. 2014;13:28-35. 